\title{
Multiple Positive Solutions for Nonlinear Fractional Differential Equations with Integral Boundary Value Conditions and a Parameter
}

\author{
Juan Mao $\mathbb{D}^{1}$ and Daliang Zhao $\mathbb{D}^{2}$ \\ ${ }^{1}$ Department of Basic Courses, Shandong Polytechnic, Jinan, 250104, China \\ ${ }^{2}$ School of Mathematics and Statistics, Shandong Normal University, Jinan, 250014, China \\ Correspondence should be addressed to Daliang Zhao; dlzhao928@sdnu.edu.cn
}

Received 20 January 2019; Accepted 28 March 2019; Published 2 May 2019

Academic Editor: Pasquale Vetro

Copyright (c) 2019 Juan Mao and Daliang Zhao. This is an open access article distributed under the Creative Commons Attribution License, which permits unrestricted use, distribution, and reproduction in any medium, provided the original work is properly cited.

By using the theory of fixed-point index on cone for differentiable operators, spectral radii of some related linear integral operators, and properties of Green's function, the existence of multiple positive solutions to a nonlinear fractional differential system with integral boundary value conditions and a parameter is established. At last, some examples are also provided to illustrate the validity of our main results.

\section{Introduction}

In recent years, the interest in the study of fractional differential equations has been growing rapidly since it has many applications in biology, mechanics, electrochemistry, and dynamical processes in self-similar structures, etc.; see [1-3], for instance. Fractional differential equations also serve as an excellent tool for the description of hereditary properties of various materials and processes. Fractionalorder models have proved to be more accurate than integer order models and have more applications. In consequence, many meaningful results in these fields have been obtained. For details, see [4-14] and references therein.

In addition, several new types of fractional derivatives are also investigated in some excellent papers. In [15], Yang studied a class of fractional derivatives of constant and variable orders for the first time. In [16], the authors introduced a new fractional derivative without singular kernel, and this was an extension of the Riemann-Liouville fractional derivative with singular kernel and had some important applications in the modeling of the fractional-order heat flow.

Meanwhile, there are a lot of papers (see [3, 17-28], for instance) dealing with the existence of positive solutions of nonlinear fractional differential equations by use of fixedpoint theorems, monotone iterative technique, and upper and lower solution method. For example, in [25], by using the method of upper and lower solutions and Schauder fixed theorem, Vong investigated the positive solutions for the following nonlocal fractional BVP:

$$
\begin{aligned}
{ }^{C} D_{0^{+}}^{\alpha} u(t)+f(t, u(t)) & =0, \quad 0<t<1, \\
u^{\prime}(0) & =\cdots=u^{(n-1)}(0)=0, \\
u(1) & =\int_{0}^{1} u(s) d \mu(s),
\end{aligned}
$$

where $n \geq 2, \alpha \in(n-1, n)$, and $\mu(s)$ is a function of bounded variation. $f$ may be singular at $t=1$.

In [26], The authors studied a class of higher order fractional BVP. In view of monotone iterative method, they got an existence and uniqueness result of positive solution.

Goodrich [8] dealt with a problem similar to the system in [26] but with local conditions. By deriving properties of the Green's function and by means of Guo-Krasnoselskii's fixedpoint theorem, the author established some existence results of at least one positive solution provided that $f(t, x)$ satisfies some growth conditions.

However, compared with the methods of fixed-point theorems, Leray-Schauder theory, and monotone iterative 
technique, there is little work investigating positive solution of fractional integral boundary value problem by use of the differentiable operator method. On the other hand, the results about such system with a parameter are relatively scarce.

Motivated by all the above-mentioned works, we aim to establish some existence criteria of multiple positive solutions for the following nonlinear fractional BVP with integral boundary conditions and a parameter:

$$
\begin{aligned}
D_{0^{+}}^{\alpha} u(t)+f(t, u(t)) & =0, \quad t \in(0,1), \\
u^{(j)}(0) & =0, \quad 0 \leq j \leq 3, \quad j \neq 1, \\
u^{\prime}(1) & =\lambda \int_{0}^{\xi} u(s) d s,
\end{aligned}
$$

where $3<\alpha \leq 4,0 \leq \lambda<\alpha, 0<\xi \leq 1,0 \leq$ $\lambda \xi^{2}<2, D_{0^{+}}^{\alpha}$ is the Caputo fractional derivative, and $f$ : $[0,1] \times[0,+\infty) \longrightarrow[0,+\infty)$ is continuous. It is worth mentioning that there is no paper studying the multiple positive solutions for the fractional differential systems by using the differentiable operator method. Besides, to the best of our knowledge, no contribution exists investigating the existence of positive solutions for BVP (2). It should be noted that $\xi \in(0,1]$ is more general than those in $[23,25,26]$, in which $\xi$ equals one.

Contributions of this article are as follows. Firstly, we derive the corresponding Green's function for fractional BVP (2) and argue its important properties which will play an important role in our proof. Also, this is the key to establish an appropriate cone. Secondly, by using the differential operator method, spectrum theory, and the properties of Green's function, we firstly investigate some existence results of multiple positive solutions for the considered fractional system. The methods used in the present paper are different from all the above-mentioned works.

This paper is organized in the following way. In Section 2, we give some necessary preliminaries and lemmas. Section 3 establishes sufficient conditions for the existence of multiple positive solutions to system (2). In Section 4, some examples are given to highlight the value of the new contributions.

\section{Preliminaries and Some Lemmas}

We now recall some results about fractional calculus in common use. These materials can be found in the recent literature; see $[2,3,29,30]$.
Definition 1. The Riemann-Liouville fractional integral of order $\alpha \in(3,4]$ of a continuous function $u:(0,+\infty) \longrightarrow \mathbb{R}$ is given by

$$
I_{0^{+}}^{\alpha} u(t)=\frac{1}{\Gamma(\alpha)} \int_{0}^{t}(t-s)^{\alpha-1} u(s) d s,
$$

provided that the right side integral is pointwise defined on $(0,+\infty)$.

Definition 2. The Caputo fractional derivative of order $\alpha \epsilon$ $(3,4]$ of a continuous function $u:(0, \infty) \longrightarrow R$ is given by

$$
{ }^{C} D_{0^{+}}^{\alpha} u(t)=\frac{1}{\Gamma(4-\alpha)} \int_{0}^{t} \frac{u^{(4)}(s)}{(t-s)^{\alpha-3}} d s
$$

provided that the right side integral is pointwise defined on $[0, \infty)$.

Lemma 3. Let $3<\alpha \leq 4$. Then

$$
I_{0^{+}}^{\alpha C} D_{0^{+}}^{\alpha} u(t)=u(t)+c_{0}+c_{1} t+c_{2} t^{2}+c_{3} t^{3}
$$

for some $c_{i} \in R, i=0,1,2,3$.

Let $E=C[0,1]$ be endowed with the norm $\|u\|=$ $\max \{|u(t)|: t \in[0,1]\}$ for each $u \in E$. Clearly, $(E,\|\cdot\|)$ is a Banach space.

Lemma 4. Suppose function $x \in C[0,1]$. Then the unique solution of the following system

$$
\begin{aligned}
D_{0^{+}}^{\alpha} u(t)+x(t) & =0, \quad t \in(0,1), \\
u^{(j)}(0) & =0, \quad 0 \leq j \leq 3, \quad j \neq 1, \\
u^{\prime}(1) & =\lambda \int_{0}^{\xi} u(s) d s,
\end{aligned}
$$

where $3<\alpha \leq 4,0 \leq \lambda<\alpha, 0<\xi \leq 1,0 \leq \lambda \xi^{2}<2$, is given by

$$
u(t)=\int_{0}^{1} G(t, s) x(s) d s
$$

where $G(t, s)$ is the Green's function of system (6) given by

$$
G(t, s)= \begin{cases}\frac{(\alpha-1) t(1-s)^{\alpha-2}-(\lambda / \alpha)(\xi-s)^{\alpha} t-\left(1-\lambda \xi^{2} / 2\right)(t-s)^{\alpha-1}}{\left(1-\lambda \xi^{2} / 2\right) \Gamma(\alpha)}, & 0 \leq s \leq \min \{\xi, t\}, \\ \frac{(\alpha-1) t(1-s)^{\alpha-2}-\left(1-\lambda \xi^{2} / 2\right)(t-s)^{\alpha-1}}{\left(1-\lambda \xi^{2} / 2\right) \Gamma(\alpha)}, & 0 \leq \xi \leq s \leq t \leq 1, \\ \frac{(\alpha-1) t(1-s)^{\alpha-2}-(\lambda / \alpha)(\xi-s)^{\alpha} t}{\left(1-\lambda \xi^{2} / 2\right) \Gamma(\alpha)}, & 0 \leq t \leq s \leq \xi \leq 1, \\ \left(1-\lambda \xi^{2} / 2\right) \Gamma(\alpha) & \max \{\xi, t\} \leq s \leq 1 .\end{cases}
$$


Proof. From Lemma 3, it follows that

$$
\begin{aligned}
u(t) & =-I_{0^{+}}^{\alpha} x(t)+c_{0}+c_{1} t+c_{2} t^{2}+c_{3} t^{3} \\
& =-\int_{0}^{t} \frac{(t-s)^{\alpha-1}}{\Gamma(\alpha)} x(s) d s+c_{0}+c_{1} t+c_{2} t^{2}+c_{3} t^{3},
\end{aligned}
$$

where $c_{i} \in R, i=0,1,2,3$.

So, one has

$$
\begin{aligned}
& u^{\prime}(t)=-\int_{0}^{t} \frac{(t-s)^{\alpha-2}}{\Gamma(\alpha-1)} x(s) d s+c_{1}+2 c_{2} t+3 c_{3} t^{2}, \\
& u^{\prime \prime}(t)=-\int_{0}^{t} \frac{(t-s)^{\alpha-3}}{\Gamma(\alpha-2)} x(s) d s+2 c_{2}+6 c_{3} t, \\
& u^{\prime \prime \prime}(t)=-\int_{0}^{t} \frac{(t-s)^{\alpha-4}}{\Gamma(\alpha-3)} x(s) d s+6 c_{3} .
\end{aligned}
$$

From $u^{(j)}(0)=0,0 \leq j \leq 3, j \neq 1, u^{\prime}(1)=\lambda \int_{0}^{\xi} u(s) d s$, we get

$$
\begin{aligned}
& c_{0}=c_{2}=c_{3}=0, \\
& c_{1}=\int_{0}^{1} \frac{(1-s)^{\alpha-2}}{\Gamma(\alpha-1)} x(s) d s+\lambda \int_{0}^{\xi} u(s) d s .
\end{aligned}
$$

So we have,

$$
\begin{aligned}
u^{\prime}(1)= & -\int_{0}^{1} \frac{(1-s)^{\alpha-2}}{\Gamma(\alpha-1)} x(s) d s \\
& +\left(\int_{0}^{1} \frac{(1-s)^{\alpha-2}}{\Gamma(\alpha-1)} x(s) d s+\lambda \int_{0}^{\xi} u(s) d s\right), \\
u(t)=- & \int_{0}^{t} \frac{(t-s)^{\alpha-1}}{\Gamma(\alpha)} x(s) d s \\
& +t\left(\int_{0}^{1} \frac{(1-s)^{\alpha-2}}{\Gamma(\alpha-1)} x(s) d s+\lambda \int_{0}^{\xi} u(s) d s\right) .
\end{aligned}
$$

By direct integration to (13), it is easy to get

$$
\begin{aligned}
\int_{0}^{\xi} u(s) d s & \\
= & -\frac{1}{\Gamma(\alpha)} \int_{0}^{\xi} \int_{0}^{x}(x-s)^{\alpha-1} x(s) d s d x \\
& +\int_{0}^{\xi} s d s\left(\int_{0}^{1} \frac{(1-s)^{\alpha-2}}{\Gamma(\alpha-1)} x(s) d s+\lambda \int_{0}^{\xi} u(s) d s\right) \\
= & -\frac{1}{\Gamma(\alpha)} \int_{0}^{\xi} \frac{(\xi-\alpha)^{\alpha}}{\alpha} x(s) d s \\
& +\frac{\xi^{2}}{2}\left(\int_{0}^{1} \frac{(1-s)^{\alpha-2}}{\Gamma(\alpha-1)} x(s) d s+\lambda \int_{0}^{\xi} u(s) d s\right) .
\end{aligned}
$$

Through the conditions $u^{\prime}(1)=\lambda \int_{0}^{\xi} u(s) d s$, (12), and (14), one has

$$
\begin{aligned}
-\int_{0}^{1} & \frac{(1-s)^{\alpha-2}}{\Gamma(\alpha-1)} x(s) d s \\
+ & \left(\int_{0}^{1} \frac{(1-s)^{\alpha-2}}{\Gamma(\alpha-1)} x(s) d s+\lambda \int_{0}^{\xi} u(s) d s\right) \\
= & -\frac{\lambda}{\Gamma(\alpha)} \int_{0}^{\xi} \frac{(\xi-\alpha)^{\alpha}}{\alpha} x(s) d s \\
& +\frac{\lambda \xi^{2}}{2}\left(\int_{0}^{1} \frac{(1-s)^{\alpha-2}}{\Gamma(\alpha-1)} x(s) d s+\lambda \int_{0}^{\xi} u(s) d s\right) .
\end{aligned}
$$

Then, we obtain

$$
\begin{gathered}
\int_{0}^{1} \frac{(1-s)^{\alpha-2}}{\Gamma(\alpha-1)} x(s) d s+\lambda \int_{0}^{\xi} u(s) d s \\
=\int_{0}^{1} \frac{(1-s)^{\alpha-2}}{\left(1-\lambda \xi^{2} / 2\right) \Gamma(\alpha-1)} x(s) d s \\
\quad-\int_{0}^{\xi} \frac{\lambda(\xi-s)^{\alpha}}{\alpha\left(1-\lambda \xi^{2} / 2\right) \Gamma(\alpha)} x(s) d s .
\end{gathered}
$$

Therefore, we can get the following solution of (6) by (13),

$$
\begin{aligned}
u(t)= & -\int_{0}^{t} \frac{(t-s)^{\alpha-1}}{\Gamma(\alpha)} x(s) d s \\
& +\int_{0}^{1} \frac{(1-s)^{\alpha-2} t}{\left(1-\lambda \xi^{2} / 2\right) \Gamma(\alpha-1)} x(s) d s \\
& -\int_{0}^{\xi} \frac{(\lambda / \alpha)(\xi-s)^{\alpha} t}{\left(1-\lambda \xi^{2} / 2\right) \Gamma(\alpha)} x(s) d s .
\end{aligned}
$$

In the following, we divide the proof into two cases.

Case $1(t \leq \xi)$

$$
u(t)=-\int_{0}^{t} \frac{(t-s)^{\alpha-1}}{\Gamma(\alpha)} x(s) d s
$$

$$
\begin{aligned}
& +\left(\int_{0}^{t}+\int_{t}^{\xi}+\int_{\xi}^{1}\right) \frac{(1-s)^{\alpha-2} t}{\left(1-\lambda \xi^{2} / 2\right) \Gamma(\alpha-1)} x(s) d s \\
& -\left(\int_{0}^{t}+\int_{t}^{\xi}\right) \frac{(\lambda / \alpha)(\xi-s)^{\alpha} t}{\left(1-\lambda \xi^{2} / 2\right) \Gamma(\alpha)} x(s) d s \\
& =\int_{0}^{t} \frac{(\alpha-1) t(1-s)^{\alpha-2}-(\lambda / \alpha)(\xi-s)^{\alpha} t-\left(1-\lambda \xi^{2} / 2\right)(t-s)^{\alpha-1}}{\left(1-\lambda \xi^{2} / 2\right) \Gamma(\alpha)} x(s) d s \\
& +\int_{t}^{\xi} \frac{(\alpha-1) t(1-s)^{\alpha-2}-(\lambda / \alpha)(\xi-s)^{\alpha} t}{\left(1-\lambda \xi^{2} / 2\right) \Gamma(\alpha)} x(s) d s \\
& +\int_{\xi}^{1} \frac{(\alpha-1) t(1-s)^{\alpha-2}}{\left(1-\lambda \xi^{2} / 2\right) \Gamma(\alpha)} x(s) d s \\
& =\int_{0}^{1} G(t, s) x(s) d s .
\end{aligned}
$$


Case $2(t \geq \xi)$

$$
\begin{aligned}
u(t) & =-\left(\int_{0}^{\xi}+\int_{\xi}^{t}\right) \frac{(t-s)^{\alpha-1}}{\Gamma(\alpha)} x(s) d s \\
& +\left(\int_{0}^{\xi}+\int_{\xi}^{t}+\int_{t}^{1}\right) \frac{(1-s)^{\alpha-2} t}{\left(1-\lambda \xi^{2} / 2\right) \Gamma(\alpha-1)} x(s) d s \\
& -\int_{0}^{\xi} \frac{(\lambda / \alpha)(\xi-s)^{\alpha} t}{\left(1-\lambda \xi^{2} / 2\right) \Gamma(\alpha)} x(s) d s \\
& =\int_{0}^{\xi} \frac{(\alpha-1) t(1-s)^{\alpha-2}-(\lambda / \alpha)(\xi-s)^{\alpha} t-\left(1-\lambda \xi^{2} / 2\right)(t-s)^{\alpha-1}}{\left(1-\lambda \xi^{2} / 2\right) \Gamma(\alpha)} x(s) d s \\
& +\int_{\xi}^{t} \frac{(\alpha-1) t(1-s)^{\alpha-2}-\left(1-\lambda \xi^{2} / 2\right)(t-s)^{\alpha-1}}{\left(1-\lambda \xi^{2} / 2\right) \Gamma(\alpha)} x(s) d s \\
& +\int_{t}^{1} \frac{(\alpha-1) t(1-s)^{\alpha-2}}{\left(1-\lambda \xi^{2} / 2\right) \Gamma(\alpha)} x(s) d s \\
& =\int_{0}^{1} G(t, s) x(s) d s .
\end{aligned}
$$

The conclusion of this lemma follows

Next, we give some important properties about Green's function $G(t, s)$ belonging to system (6).

Lemma 5. The Green's function $G(t, s)$ of system (6) satisfies the following inequalities:

(i) $G(t, s) \leq\left((\alpha-1) /\left(1-\lambda \xi^{2} / 2\right) \Gamma(\alpha)\right) t(1-s)^{\alpha-2}, \forall t, s \in$ $[0,1]$;

(ii) $G(t, s) \leq\left((\alpha-1) /\left(1-\lambda \xi^{2} / 2\right) \Gamma(\alpha)\right)(1-s)^{\alpha-2}, \forall t, s \in$

(iii) $G(t, s) \geq\left(\left(\alpha-1-\lambda / \alpha-\left(1-\lambda \xi^{2} / 2\right)\right) /(1-\right.$ $\left.\left.\lambda \xi^{2} / 2\right) \Gamma(\alpha)\right) t(1-s)^{\alpha-2}, \forall t, s \in(0,1)$;

(iv) $G(t, s)>0, \forall t, s \in(0,1)$.

Proof. We denote $Q(s)=\left(\left(\alpha-1-\lambda / \alpha-\left(1-\lambda \xi^{2} / 2\right)\right) /(1-\right.$ $\left.\left.\lambda \xi^{2} / 2\right) \Gamma(\alpha)\right)(1-s)^{\alpha-2}$ and take $H(t, s)=G(t, s) / Q(s)$.

For $0 \leq s \leq \min \{\xi, t\}<1$, one has

$$
\begin{aligned}
& H(1, s) \\
& =\frac{(\alpha-1)(1-s)^{\alpha-2}-(\lambda / \alpha)(\xi-s)^{\alpha}-\left(1-\lambda \xi^{2} / 2\right)(1-s)^{\alpha-1}}{\left(\alpha-1-\lambda / \alpha-\left(1-\lambda \xi^{2} / 2\right)\right)(1-s)^{\alpha-2}} \\
& >\frac{(\alpha-1)(1-s)^{\alpha-2}-(\lambda / \alpha)(1-s)^{\alpha-2}-\left(1-\lambda \xi^{2} / 2\right)(1-s)^{\alpha-2}}{\left(\alpha-1-\lambda / \alpha-\left(1-\lambda \xi^{2} / 2\right)\right)(1-s)^{\alpha-2}}
\end{aligned}
$$$$
=1 \text {, }
$$

$$
\begin{gathered}
H(s, s)=\frac{(\alpha-1) s(1-s)^{\alpha-2}-(\lambda / \alpha)(\xi-s)^{\alpha} s}{\left(\alpha-1-\lambda / \alpha-\left(1-\lambda \xi^{2} / 2\right)\right)(1-s)^{\alpha-2}} \\
>\frac{(\alpha-1-\lambda / \alpha)(1-s)^{\alpha-2} s}{\left(\alpha-1-\lambda / \alpha-\left(1-\lambda \xi^{2} / 2\right)\right)(1-s)^{\alpha-2}}>s,
\end{gathered}
$$

and

$$
\begin{aligned}
\frac{\partial^{2} H}{\partial t^{2}}(t, s) & =-\frac{\left(1-\lambda \xi^{2} / 2\right)(\alpha-1)(\alpha-2)(t-s)^{\alpha-3}}{\left(\alpha-1-\lambda / \alpha-\left(1-\lambda \xi^{2} / 2\right)\right)(1-s)^{\alpha-2}} \\
& \leq 0 .
\end{aligned}
$$

This means that $H(\cdot, s)$ is concave on $[\mathrm{s}, 1]$. So we get $H(t, s) \geq$ $t$.
For $0 \leq \xi \leq s \leq t<1$, we have

$$
\begin{aligned}
H(1, s) & =\frac{(\alpha-1)(1-s)^{\alpha-2}-\left(1-\lambda \xi^{2} / 2\right)(1-s)^{\alpha-1}}{\left(\alpha-1-\lambda / \alpha-\left(1-\lambda \xi^{2} / 2\right)\right)(1-s)^{\alpha-2}} \\
& >\frac{\left(\alpha-1-\left(1-\lambda \xi^{2} / 2\right)\right)(1-s)^{\alpha-2}}{\left(\alpha-1-\lambda / \alpha-\left(1-\lambda \xi^{2} / 2\right)\right)(1-s)^{\alpha-2}} \\
& >1, \\
H(s, s) & =\frac{(\alpha-1) s(1-s)^{\alpha-2}}{\left(\alpha-1-\lambda / \alpha-\left(1-\lambda \xi^{2} / 2\right)\right)(1-s)^{\alpha-2}} \\
& >s,
\end{aligned}
$$

and

$$
\begin{aligned}
\frac{\partial^{2} H}{\partial t^{2}}(t, s) & =-\frac{\left(1-\lambda \xi^{2} / 2\right)(\alpha-1)(\alpha-2)(t-s)^{\alpha-3}}{\left(\alpha-1-\lambda / \alpha-\left(1-\lambda \xi^{2} / 2\right)\right)(1-s)^{\alpha-2}} \\
& \leq 0
\end{aligned}
$$

These three facts can also indicate $H(t, s) \geq t$.

For $0 \leq t \leq s<\xi \leq 1$, we have $H(0, s)=0$, and

$$
\begin{aligned}
H(s, s) & =\frac{\left((\alpha-1)(1-s)^{\alpha-2}-(\lambda / \alpha)(\xi-s)^{\alpha}\right) s}{\left(\alpha-1-\lambda / \alpha-\left(1-\lambda \xi^{2} / 2\right)\right)(1-s)^{\alpha-2}} \\
& >\frac{(\alpha-1-\lambda / \alpha)(1-s)^{\alpha-2} s}{\left(\alpha-1-\lambda / \alpha-\left(1-\lambda \xi^{2} / 2\right)\right)(1-s)^{\alpha-2}} \\
& >s, \\
\frac{\partial H}{\partial t}(t, s) & =\frac{(\alpha-1)(1-s)^{\alpha-2}-(\lambda / \alpha)(\xi-s)^{\alpha}}{\left(\alpha-1-\lambda / \alpha-\left(1-\lambda \xi^{2} / 2\right)\right)(1-s)^{\alpha-2}} \\
& >\frac{(\alpha-1-\lambda / \alpha)(1-s)^{\alpha-2}}{\left(\alpha-1-\lambda / \alpha-\left(1-\lambda \xi^{2} / 2\right)\right)(1-s)^{\alpha-2}}
\end{aligned}
$$$$
>1 \text {. }
$$

So, we can get that $H(t, s) \geq t$.

For $\max \{\xi, t\} \leq s<1$, we obtain $H(0, s)=0$, and

$$
\begin{aligned}
H(s, s) & =\frac{(\alpha-1)(1-s)^{\alpha-2} s}{\left(\alpha-1-\lambda / \alpha-\left(1-\lambda \xi^{2} / 2\right)\right)(1-s)^{\alpha-2}} \\
& >s, \\
\frac{\partial H}{\partial t}(t, s) & =\frac{(\alpha-1)(1-s)^{\alpha-2}}{\left(\alpha-1-\lambda / \alpha-\left(1-\lambda \xi^{2} / 2\right)\right)(1-s)^{\alpha-2}}
\end{aligned}
$$

$>1$.

Hence, we also have $H(t, s) \geq t$.

Consequently, one gets the validity of (iii) and (iv). The establishment of $(i)$ and $(i i)$ is obvious.

Lemma 6 (see [29]). Let $X$ be a Banach space, $P$ be a cone in $X$, and $\Omega(P)$ be a bounded open subset in P. Suppose that 
$A: \overline{\Omega(P)} \longrightarrow P$ is a completely continuous operator. Then the following results hold:

(i) if there exists $u_{0} \in P \backslash\{\theta\}$ such that $u \neq A u+\lambda u_{0}$, for any $u \in \partial \Omega(P), \lambda \geq 0$, then $i(A, \Omega(P), P)=0$;

(ii) if $\theta \in \Omega(P), A u \neq \lambda u$, for any $u \in \partial \Omega(P), \lambda \geq 1$, then $i(A, \Omega(P), P)=1$.

Lemma 7 (see [29]). Let $P$ be a cone in a Banach space $E, A$ : $P \longrightarrow P$ be completely continuous, and $A \theta=\theta$. Suppose that $A$ is differentiable at $\theta$ along $P$ and 1 is not an eigenvalue of $A_{+}^{\prime}(\theta)$ corresponding to a positive eigenvector. Moreover, if $A_{+}^{\prime}(\theta)$ has no positive eigenvectors corresponding to an eigenvalue greater than one, then there exists $r_{0}>0$ such that $i\left(A, P_{r}, P\right)=1$, for $0<r \leq r_{0}$, where $P_{r}=\{x \in P:\|u\|<r\}$.

Lemma 8 (see [29]). Let $P$ be a cone in a Banach space $E$ and $A: P \longrightarrow P$ be completely continuous. Suppose that $A$ is differentiable at $\infty$ along $P$ and 1 is not an eigenvalue of $A_{+}^{\prime}(\infty)$ corresponding to a positive eigenvector. Moreover, if $A_{+}^{\prime}(\infty)$ has no positive eigenvectors corresponding to an eigenvalue greater than one, then there exists $R_{0}>0$ such that $i\left(A, P_{R}, P\right)=1$, for $R \geq R_{0}$, where $P_{R}=\{x \in P:\|u\|<R\}$.

Define a cone $P$ by

$$
P=\{u \in E: u(t) \geq M t\|u\|, t \in[0,1]\},
$$

where $M=\left(\alpha-1-\lambda / \alpha-\left(1-\lambda \xi^{2} / 2\right)\right) /(\alpha-1)$. Let $P_{r}=\{u \in$ $P:\|u\|<r\}(r>0)$.

Define integral operators $L$ and $T$ by

$$
\begin{aligned}
& L u(t)=\int_{0}^{1} G(t, s) u(s) d s, \quad u \in E ; \\
& T u(t)=\int_{0}^{1} G(t, s) f(s, u(s)) d s, \quad u \in P .
\end{aligned}
$$

Lemma 9 (see [14]). $L: P \longrightarrow P$ is completely continuous and the spectral radius $r(L)>0$.

By a similar process in the proof of Lemma 9, we have the following lemma.

Lemma 10. Let $f \in C([0,1] \times[0,+\infty),[0,+\infty))$, and then operator T maps cone $P$ into $P$ and $T$ is completely continuous.

\section{Main Results}

For convenience in the following discussion, we present the following notations and assumptions:

$$
\begin{aligned}
f_{\infty} & =\liminf _{u \longrightarrow+\infty} \min _{t \in[0,1]} \frac{f(t, u)}{u}, \\
f_{0} & =\liminf _{u \longrightarrow 0^{+}} \min _{t \in[0,1]} \frac{f(t, u)}{u}, \\
f^{\infty} & =\limsup _{u \longrightarrow+\infty} \max _{t \in[0,1]} \frac{f(t, u)}{u}, \\
f^{0} & =\limsup _{u \longrightarrow 0^{+}} \max _{t \in[0,1]} \frac{f(t, u)}{u} .
\end{aligned}
$$

(H1) $f(t, 0)=0, f_{u} \in C([0,1] \times[0,+\infty))$, and $f_{u}(t, 0)>0$, $\forall t \in[0,1]$.

(H2) $\int_{0}^{1}(1-t)^{\alpha-2} f_{u}(t, 0) d t<\left(1-\lambda \xi^{2} / 2\right) \Gamma(\alpha) /(\alpha-1)$.

(H3) There exists $\phi \in C([0,1],[0,+\infty)), \phi \not \equiv 0$, such that $\lim _{u \longrightarrow+\infty}(f(t, u) / u)=\phi(t)$ uniformly holds with respect to $t$ on $[0,1]$, and $\int_{0}^{1}(1-t)^{\alpha-2} \phi(t) d t<(1-$ $\left.\lambda \xi^{2} / 2\right) \Gamma(\alpha) /(\alpha-1)$.

Adopting regular approaches similar to that in [14], we give the following two lemmas.

Lemma 11. Suppose that (H1) and (H2) hold. Thus $T$ is differentiable at $\theta$ along $P, T \theta=\theta$, and

$$
T_{+}^{\prime}(\theta) u=\int_{0}^{1} G(t, s) f_{u}(s, 0) u(s) d s, \quad u \in P .
$$

In addition, $T_{+}^{\prime}(\theta)$ has no positive eigenvectors corresponding to an eigenvalue greater than or equal to one.

Proof. Obviously, $T \theta=\theta$ by $f(t, 0)=0$. From mean value theorem, it follows that

$$
f(t, u)=f(t, u)-f(t, 0)=f_{u}\left(t, u_{0}\right) u,
$$

for some $u_{0} \in(0, u)$. Notice $f_{u} \in C([0,1] \times[0,+\infty))$, and we can obtain that for any $\varepsilon>0$, there exists a $\delta>0$ such that $0<u_{0}<u<\delta$ and

$$
\begin{aligned}
\left|f(t, u)-f_{u}(t, 0) u\right| \leq & \left|f(t, u)-f_{u}\left(t, u_{0}\right) u\right| \\
& +\left|f_{u}\left(t, u_{0}\right)-f_{u}(t, 0)\right| u \\
& =\left|f_{u}\left(t, u_{0}\right)-f_{u}(t, 0)\right| u \\
& <\left(1-\frac{\lambda \xi^{2}}{2}\right) \Gamma(\alpha) \varepsilon u .
\end{aligned}
$$

So for any $u \in P,\|u\|<\delta$, noticing (31) and Lemma 5, we get

$$
\begin{aligned}
& \left|T u(t)-\int_{0}^{1} G(t, s) f_{u}(s, 0) u(s) d s\right| \\
& \quad \leq \int_{0}^{1} G(t, s)\left|f(s, u)-f_{u}(s, 0)\right| d s \\
& \quad \leq\left(1-\frac{\lambda \xi^{2}}{2}\right) \Gamma(\alpha) \varepsilon\|u\| \int_{0}^{1} G(t, s) d s \leq \varepsilon\|u\|,
\end{aligned}
$$

which means that

$$
T_{+}^{\prime}(\theta) u=\int_{0}^{1} G(t, s) f_{u}(s, 0) u(s) d s, \quad u \in P .
$$

Now, we are ready to show that $T_{+}^{\prime}(\theta)$ has no positive eigenvectors corresponding to an eigenvalue greater than or equal to one. Otherwise, there exist $u^{*} \in P \backslash\{\theta\}$ and $\lambda^{*} \geq 1$ such that $T_{+}^{\prime}(\theta) u^{*}=\lambda^{*} u^{*}$. Then

$$
\begin{aligned}
& u^{*}(t) \leq \lambda^{*} u^{*}(t)=\int_{0}^{1} G(t, s) f_{u}(s, 0) u^{*}(s) d s \\
& \quad \leq \frac{\alpha-1}{\left(1-\lambda \xi^{2} / 2\right) \Gamma(\alpha)} \int_{0}^{1}(1-s)^{\alpha-2} f_{u}(s, 0) u^{*}(s) d s .
\end{aligned}
$$


Notice $f_{u}(t, 0)>0$, and it follows

$$
\begin{aligned}
& (1-t)^{\alpha-2} f_{u}(t, 0) u^{*}(t) \\
& \leq \frac{\alpha-1}{\left(1-\lambda \xi^{2} / 2\right) \Gamma(\alpha)}(1-t)^{\alpha-2} f_{u}(t, 0) \\
& \quad \cdot \int_{0}^{1}(1-s)^{\alpha-2} f_{u}(s, 0) u^{*}(s) d s .
\end{aligned}
$$

By direct integration to $(35)$ on $[0,1]$, we obtain

$$
\begin{aligned}
& \int_{0}^{1}(1-t)^{\alpha-2} f_{u}(t, 0) u^{*}(t) d t \leq \frac{\alpha-1}{\left(1-\lambda \xi^{2} / 2\right) \Gamma(\alpha)} \\
& \quad \cdot \int_{0}^{1}(1-t)^{\alpha-2} f_{u}(t, 0) d t \\
& \quad \cdot \int_{0}^{1}(1-s)^{\alpha-2} f_{u}(s, 0) u^{*}(s) d s .
\end{aligned}
$$

Notice $\int_{0}^{1}(1-t)^{\alpha-2} f_{u}(t, 0) u^{*}(t) d t>0$, from which we infer that

$$
\frac{\alpha-1}{\left(1-\lambda \xi^{2} / 2\right) \Gamma(\alpha)} \int_{0}^{1}(1-t)^{\alpha-2} f_{u}(t, 0) d t \geq 1,
$$

which contradicts (H2). The proof is thus completed.

Lemma 12. Suppose that (H3) holds. Thus $T$ is differentiable at $\infty$ along $P$ and

$$
T_{+}^{\prime}(\infty) u=\int_{0}^{1} G(t, s) \phi(s) u(s) d s, \quad u \in P .
$$

In addition, $T_{+}^{\prime}(\infty)$ has no positive eigenvectors corresponding to an eigenvalue greater than or equal to one.

Proof. From (H3), it follows that

$$
|f(t, u)-\phi(t) u|<C+\varepsilon u, \quad \forall u \geq 0, t \in[0,1],
$$

where $\varepsilon>0$, and $C$ is a constant corresponding to $\varepsilon$.

Thus, for any $u \in P, t \in[0,1]$, it has

$$
\begin{aligned}
& \left|T u(t)-\int_{0}^{1} G(t, s) \phi(s) u(s) d s\right| \\
& \quad \leq \int_{0}^{1} G(t, s)|f(s, u(s))-\phi(s) u(s)| d s \\
& \quad \leq(C+\varepsilon\|u\|) \int_{0}^{1} G(t, s) d s \leq \frac{C+\varepsilon\|u\|}{\left(1-\lambda \xi^{2} / 2\right) \Gamma(\alpha)},
\end{aligned}
$$

which implies that

$$
\begin{aligned}
& \frac{\left|T u(t)-\int_{0}^{1} G(t, s) \phi(s) u(s) d s\right|}{\|u\|} \\
& \quad \leq \frac{C}{\left(1-\lambda \xi^{2} / 2\right) \Gamma(\alpha)\|u\|}+\frac{\varepsilon}{\left(1-\lambda \xi^{2} / 2\right) \Gamma(\alpha)} \\
& \quad<\frac{2}{\left(1-\lambda \xi^{2} / 2\right) \Gamma(\alpha)} \varepsilon,
\end{aligned}
$$

when $\|u\|>C / \varepsilon$. Therefore, $T_{+}^{\prime}(\infty) u=\int_{0}^{1} G(t, s) \phi(s) u(s) d s$, $u \in P$.

Suppose that $T_{+}^{\prime}(\infty)$ has positive eigenvectors corresponding to an eigenvalue greater than or equal to one. Then we can choose a function $u^{*} \in P \backslash\{\theta\}$ and a constant $\lambda^{*} \geq 1$ satisfying $T_{+}^{\prime}(\theta) u^{*}=\lambda^{*} u^{*}$, and thus

$$
\begin{aligned}
& u^{*}(t) \leq \lambda^{*} u^{*}(t)=\int_{0}^{1} G(t, s) \phi(s) u^{*}(s) d s \\
& \quad \leq \frac{\alpha-1}{\left(1-\lambda \xi^{2} / 2\right) \Gamma(\alpha)} \int_{0}^{1}(1-s)^{\alpha-2} \phi(s) u^{*}(s) d s .
\end{aligned}
$$

By means of $\phi \in C([0,1],[0,+\infty)), \phi \not \equiv 0$, one has

$$
\begin{aligned}
& (1-t)^{\alpha-2} \phi(t) u^{*}(t) \leq \frac{\alpha-1}{\left(1-\lambda \xi^{2} / 2\right) \Gamma(\alpha)}(1-t)^{\alpha-2} \\
& \cdot \phi(t) \int_{0}^{1}(1-s)^{\alpha-2} \phi(s) u^{*}(s) d s .
\end{aligned}
$$

Integrating (43) with respect to $t$ on $[0,1]$ gives

$$
\begin{gathered}
\int_{0}^{1}(1-t)^{\alpha-2} \phi(t) u^{*}(t) d t \leq \frac{\alpha-1}{\left(1-\lambda \xi^{2} / 2\right) \Gamma(\alpha)} \\
\quad \cdot \int_{0}^{1}(1-t)^{\alpha-2} \phi(t) d t \int_{0}^{1}(1-s)^{\alpha-2} \phi(s) u^{*}(s) d s .
\end{gathered}
$$

From the condition $\int_{0}^{1}(1-t)^{\alpha-2} \phi(t) u^{*}(t) d t>0$, it follows that

$$
\frac{\alpha-1}{\left(1-\lambda \xi^{2} / 2\right) \Gamma(\alpha)} \int_{0}^{1}(1-t)^{\alpha-2} \phi(t) d t \geq 1 .
$$

Notice (H3); this is a contradict. Hence, Lemma 12 holds.

The following theorems are main results in this paper.

Theorem 13. Suppose that (H1), (H2), and $f_{\infty}>r^{-1}(L)$ hold. Then BVP (2) has at least one positive solution.

Proof. From Lemmas 9, 11, and 7, it follows that

$$
i\left(T, P_{r_{0}}, P\right)=1
$$

where $r_{0}>0$ is a suitable constant.

Let

$$
L_{n} u(t)=\int_{1 / n}^{1} G(t, s) u(s) d s, \quad n>1 .
$$

Standard arguments can show that $L_{n}: P \longrightarrow P$ is completely continuous and $r\left(L_{n}\right)>0$. Let $\lambda_{n}=r^{-1}\left(L_{n}\right), \lambda_{1}=$ $r^{-1}(L)$, and $\lim _{n \rightarrow \infty} \lambda_{n}=\lambda_{0}$. The proof of $\lambda_{0}=\lambda_{1}$ follows by arguing as in [14], so we omit the proof. Hence, there exist $N_{0}$ and $\varepsilon_{0}$ such that $\lambda_{N_{0}}<\lambda_{1}+\varepsilon_{0}$; that is

$$
r\left(L_{N_{0}}\right)>\frac{1}{r^{-1}(L)+\varepsilon_{0}} .
$$


According to Krein-Rutman theorem, we can obtain some $\psi_{N_{0}} \in E \backslash\{\theta\}$ with $\psi_{N_{0}} \geq 0$ such that

$$
\psi_{N_{0}}(t)=r^{-1}\left(L_{N_{0}}\right) \int_{1 / N_{0}}^{1} G(t, s) \psi_{N_{0}}(s) d s .
$$

Then, for all $t, \tau \in[0,1]$, one has

$$
\begin{aligned}
\psi_{N_{0}}(t) & \geq \operatorname{Mtr}^{-1}\left(L_{N_{0}}\right) \int_{1 / N_{0}}^{1} G(\tau, s) \psi_{N_{0}}(s) d s \\
& =M t \psi_{N_{0}}(\tau) .
\end{aligned}
$$

Thus, $\psi_{N_{0}}(t) \geq M t\left\|\psi_{N_{0}}\right\|$, which means $\psi_{N_{0}}(t) \in P \backslash\{\theta\}$.

Moreover, using $f_{\infty}>r^{-1}(L)$, we can choose a constant $R>0$ such that

$$
f(t, u)>\left(r^{-1}(L)+\varepsilon_{0}\right) u, \quad \forall u \geq R, t \in[0,1] .
$$

Choose $R_{0}>\max \left\{r_{0},\left(N_{0} / M\right) R\right\}$. Thus for any $u \in \partial P_{R_{0}}$, it follows

$$
u(t) \geq M t\|u\|=M t R_{0}>R, \quad t \in\left[\frac{1}{N_{0}}, 1\right] .
$$

If we can choose a function $u_{2} \in \partial P_{R_{0}}$ and a constant $\mu_{0}>$ 0 such that

$$
u_{2}(t)-T u_{2}(t)=\mu_{0} \psi_{N_{0}}(t), \quad t \in[0,1],
$$

then we know $u_{2}(t) \geq \mu_{0} \psi_{N_{0}}(t), t \in[0,1]$.

Denote

$$
\mu^{*}=\sup \left\{\mu \mid u_{2}(t) \geq \mu \psi_{N_{0}}(t), t \in[0,1]\right\} .
$$

Obviously, $0<\mu_{0} \leq \mu^{*}<+\infty$ and $u_{2}(t) \geq \mu^{*} \psi_{N_{0}}(t), t \in$ $[0,1]$. Noticing (48), (49), (51), and (53), we obtain

$$
\begin{aligned}
u_{2}(t)= & T u_{2}(t)+\mu_{0} \psi_{N_{0}}(t) \\
= & \int_{0}^{1} G(t, s) f\left(s, u_{2}(s)\right) d s+\mu_{0} \psi_{N_{0}}(t) \\
\geq & \left(r^{-1}(L)+\varepsilon_{0}\right) \int_{1 / N_{0}}^{1} G(t, s) u_{2}(s) d s \\
& +\mu_{0} \psi_{N_{0}}(t) \\
\geq & \left(r^{-1}(L)+\varepsilon_{0}\right) \mu^{*} \int_{1 / N_{0}}^{1} G(t, s) \psi_{N_{0}}(s) d s \\
& +\mu_{0} \psi_{N_{0}}(t) \\
= & \left(r^{-1}(L)+\varepsilon_{0}\right) \mu^{*} r\left(L_{N_{0}}\right) \psi_{N_{0}}(t)+\mu_{0} \psi_{N_{0}}(t) \\
> & \left(\mu^{*}+\mu_{0}\right) \psi_{N_{0}}(t), \quad t \in[0,1] .
\end{aligned}
$$

This is in contradiction with the definition of $\mu^{*}$. Then we have

$$
u(t)-T u(t) \neq \mu \psi_{N_{0}}(t),
$$$$
\forall u \in \partial P_{R_{0}}, \mu \geq 0, t \in[0,1] .
$$

Using Lemma 6 yields that $i\left(T, P_{R_{0}}, P\right)=0$. This together with (46) gives

$$
i\left(T, P_{R_{0}} \backslash \bar{P}_{r_{0}}, P\right)=i\left(T, P_{R_{0}}, P\right)-i\left(T, P_{r_{0}}, P\right)=-1 .
$$

Therefore, system (2) has at least one positive solution.

Theorem 14. Suppose that $(H 3)$ and $f_{0}>r^{-1}(L)$ hold. Then $B V P(2)$ has at least one positive solution.

Proof. Since $f_{0}>r^{-1}(L)$, we know that there exists a positive constant $r_{0}$ such that

$$
f(t, u)>r^{-1}(L) u, \quad \forall u \in\left[0, r_{0}\right], t \in[0,1] .
$$

By a similar way in Theorem 13, there exists a function $\psi(t) \epsilon$ $P \backslash\{\theta\}$ such that

$$
\psi(t)=r^{-1}(L) \int_{0}^{1} G(t, s) \psi(s) d s .
$$

Next, we are ready to show

$$
u(t)-T u(t) \neq \mu \psi(t),
$$

$$
\forall u \in \partial P_{r_{0}}, \mu \geq 0, t \in[0,1] .
$$

In fact, if not, then there exist $u_{1} \in \partial P_{r_{0}}$ and $\mu_{1}>0$ such that

$$
u_{1}(t)-T u_{1}(t)=\mu_{1} \psi(t), \quad t \in[0,1],
$$

which indicates $u_{1}(t) \geq \mu_{1} \psi(t), t \in[0,1]$.

Denote

$$
\mu^{*}=\sup \left\{\mu \mid u_{1}(t) \geq \mu \psi(t), t \in[0,1]\right\} .
$$

Obviously, $0<\mu_{1} \leq \mu^{*}<+\infty$ and $u_{1}(t) \geq \mu^{*} \psi(t)$, for any $t \in[0,1]$. This together with (58), (59), and (61) gives

$$
\begin{aligned}
u_{1}(t) & =T u_{1}(t)+\mu_{1} \psi(t) \\
& =\int_{0}^{1} G(t, s) f\left(s, u_{1}(s)\right) d s+\mu_{1} \psi(t) \\
& \geq r^{-1}(L) \int_{0}^{1} G(t, s) u_{1}(s) d s+\mu_{1} \psi(t) \\
& \geq r^{-1}(L) \mu^{*} \int_{0}^{1} G(t, s) \psi(s) d s+\mu_{1} \psi(t) \\
& =\left(\mu^{*}+\mu_{1}\right) \psi(t), \quad t \in[0,1] .
\end{aligned}
$$

By means of the definition of $\mu^{*}$, we get a contradiction. Thus, from Lemma 6, one gets

$$
i\left(T, P_{r_{0}}, P\right)=0 .
$$

One the other hand, by Lemmas 9, 12, and 8, we can choose a constant $R_{0}>r_{0}$ such that $i\left(T, P_{R_{0}}, P\right)=1$. From this and (64), we get

$$
i\left(T, P_{R_{0}} \backslash \bar{P}_{r_{0}}, P\right)=i\left(T, P_{R_{0}}, P\right)-i\left(T, P_{r_{0}}, P\right)=1 .
$$

Consequently, BVP (2) has at least one positive solution. 
Theorem 15. Suppose that (H1), (H2), and $f^{\infty}<r^{-1}(L)$ hold. In addition, suppose there exists $r>0$ such that

$$
f(t, u)>m_{0} r, \quad \forall u \in\left[\frac{M}{2} r, r\right], t \in[0,1],
$$

where $m_{0}=\left(\max _{t \in[0,1]} \int_{1 / 2}^{1} G(t, s) d s\right)^{-1}$. Then BVP (2) has at least two positive solutions.

Proof. By Lemmas 9, 11, and 7, we can choose a constant $r_{0} \in$ $(0, r)$ such that

$$
i\left(T, P_{r_{0}}, P\right)=1 \text {. }
$$

Let $\psi(t) \in P \backslash\{\theta\}$ be the eigenfunction of $L$ corresponding to $r(L)$. For $u \in \partial P_{r}$, one has $u(t) \geq t M\|u\| \geq(M / 2) r$, for any $t \in[1 / 2,1]$. Thus, it follows

$$
\begin{aligned}
T u(t) & =\int_{0}^{1} G(t, s) f(s, u(s)) d s \\
& \geq \int_{1 / 2}^{1} G(t, s) f(s, u(s)) d s \\
& >\int_{1 / 2}^{1} G(t, s) d s \cdot m_{0} r,
\end{aligned}
$$

which yields $\|T u\|>\max _{t \in[0,1]} \int_{1 / 2}^{1} G(t, s) d s \cdot m_{0} r=r$. Then, for $u \in \partial P_{r}, \mu>0$, we can obtain $\|T u+\mu \psi\| \geq\|T u\|>r$. This means $u \neq T u+\mu \psi$, for $u \in \partial P_{r}, \mu>0$. By Lemma 6 , we get

$$
i\left(T, P_{r}, P\right)=0 \text {. }
$$

On the other hand, choose $\varepsilon_{0}$ such that $0<\left(r^{-1}(L)-\right.$ $\left.\varepsilon_{0}\right)\|L\|<1$. Then by $f^{\infty}<r^{-1}(L)$, there exists $R>0$ such that

$$
f(t, u) \leq\left(r^{-1}(L)-\varepsilon_{0}\right) u, \quad u \geq R, t \in[0,1] .
$$

Let $m_{1}=\max _{(t, u) \in[0,1] \times[0, R]} f(t, u)$. Thus, we get

$$
f(t, u) \leq\left(r^{-1}(L)-\varepsilon_{0}\right) u+m_{1}, \quad \forall u \geq 0, t \in[0,1] .
$$

Choose $R_{0}>\left\{m_{1} /\left(1-\left(r^{-1}(L)-\varepsilon_{0}\right)\|L\|\right)\left(1-\lambda \xi^{2} / 2\right) \Gamma(\alpha), r\right\}$. Now, we are ready to show

$$
T u(t) \neq \mu u(t), \quad \forall u \in \partial P_{R_{0}}, \mu \geq 1, t \in[0,1] .
$$

Otherwise, suppose $u_{1} \in \partial P_{R_{0}}$ and $\mu_{1} \geq 1$ such that $T u_{1}(t)=$ $\mu_{1} u_{1}(t)$. Then,

$$
\begin{aligned}
u_{1}(t) & \leq \mu_{1} u_{1}(t)=T u_{1}(t) \\
& =\int_{0}^{1} G(t, s) f\left(s, u_{1}(s)\right) d s \\
& \leq \int_{0}^{1} G(t, s)\left(\left(r^{-1}(L)-\varepsilon_{0}\right) u_{1}(s)+m_{1}\right) d s \\
& =\left(r^{-1}(L)-\varepsilon_{0}\right) L u_{1}(t)+m_{1} \int_{0}^{1} G(t, s) d s
\end{aligned}
$$

and, noticing that $0<\left(r^{-1}(L)-\varepsilon_{0}\right)\|L\|<1$, we get that the inverse operator of $I-\left(r^{-1}(L)-\varepsilon_{0}\right) L$ exists and equals

$$
\left(I-\left(r^{-1}(L)-\varepsilon_{0}\right) L\right)^{-1}=\sum_{n=0}^{\infty}\left(r^{-1}(L)-\varepsilon_{0}\right)^{n} L^{n}
$$

from which one has $\left(I-\left(r^{-1}(L)-\varepsilon_{0}\right) L\right)^{-1}(P) \subseteq P$. This together with (73) gives

$$
u_{1}(t) \leq\left(I-\left(r^{-1}(L)-\varepsilon_{0}\right) L\right)^{-1} m_{1} \int_{0}^{1} G(t, s) d s .
$$

In addition, by $\left\|\left(I-\left(r^{-1}(L)-\varepsilon_{0}\right) L\right)^{-1}\right\| \leq 1 /\left(1-\left(r^{-1}(L)-\varepsilon_{0}\right)\|L\|\right)$ and Lemma 5 , we have

$$
\begin{aligned}
R_{0} & =\left\|u_{1}\right\| \\
& \leq\left\|\left(I-\left(r^{-1}(L)-\varepsilon_{0}\right) L\right)^{-1}\right\| \frac{m_{1}}{\left(1-\lambda \xi^{2} / 2\right) \Gamma(\alpha)} \\
& \leq \frac{m_{1}}{\left(1-\left(r^{-1}(L)-\varepsilon_{0}\right)\|L\|\right)\left(1-\lambda \xi^{2} / 2\right) \Gamma(\alpha)}<R_{0},
\end{aligned}
$$

which is a contradiction. By Lemma 6 , we get $i\left(T, P_{R_{0}}, P\right)=1$. This together with (67) and (69) guarantees that

$$
\begin{aligned}
& i\left(T, P_{r} \backslash \bar{P}_{r_{0}}, P\right)=-1, \\
& i\left(T, P_{R_{0}} \backslash \bar{P}_{r}, P\right)=1 .
\end{aligned}
$$

Therefore, BVP (2) has at least two positive solutions.

Theorem 16. Suppose that (H3) and $f^{0}<r^{-1}(L)$ hold. In addition, suppose there exists $r>0$ such that

$$
f(t, u)>M_{0} r, \quad \forall u \in[0, r], t \in[0,1],
$$

where $M_{0}=\left(\max _{t \in[0,1]} \int_{0}^{1} G(t, s) d s\right)^{-1}$. Then BVP (2) has at least two positive solutions.

Proof. Let $\psi(t) \in P \backslash\{\theta\}$ be the eigenfunction of $L$ corresponding to $r(L)$. For $u \in \partial P_{r}$, it yields

$$
\begin{aligned}
T u(t) & =\int_{0}^{1} G(t, s) f(s, u(s)) d s \\
& >\int_{0}^{1} G(t, s) d s \cdot M_{0} r,
\end{aligned}
$$

which means $\|T u\|>\max _{t \in[0,1]} \int_{0}^{1} G(t, s) d s \cdot M_{0} r=r$. Thus, we can easily get $u \neq T u+\mu \psi$, for $u \in \partial P_{r}, \mu>0$. This together with Lemma 6 gives

$$
i\left(T, P_{r}, P\right)=0 \text {. }
$$
that

By $f^{0}<r^{-1}(L)$, there exists a constant $r_{0} \in(0, r)$ such

$$
f(t, u) \leq\left(r^{-1}(L)-\varepsilon_{0}\right) u, \quad u \leq r_{0}, t \in[0,1],
$$

where $\varepsilon_{0}$ satisfies $0<\left(r^{-1}(L)-\varepsilon_{0}\right)\|L\|<1$. 
If there exist $u_{1} \in \partial P_{r_{0}}$ and $\mu_{1} \geq 1$ such that $T u_{1}(t)=$ $\mu_{1} u_{1}(t)$, we have

$$
\begin{aligned}
u_{1}(t) & \leq \mu_{1} u_{1}(t)=T u_{1}(t) \\
& =\int_{0}^{1} G(t, s) f\left(s, u_{1}(s)\right) d s \\
& \leq \int_{0}^{1} G(t, s)\left(r^{-1}(L)-\varepsilon_{0}\right) u_{1}(s) d s \\
& =\left(r^{-1}(L)-\varepsilon_{0}\right) L u_{1}(t) .
\end{aligned}
$$

Since $0<\left(r^{-1}(L)-\varepsilon_{0}\right)\|L\|<1$, we know that $I-\left(r^{-1}(L)-\varepsilon_{0}\right) L$ is reversible and

$$
\left(I-\left(r^{-1}(L)-\varepsilon_{0}\right) L\right)^{-1}=\sum_{n=0}^{\infty}\left(r^{-1}(L)-\varepsilon_{0}\right)^{n} L^{n} .
$$

By (82), one has

$$
u_{1}(t) \leq\left(I-\left(r^{-1}(L)-\varepsilon_{0}\right) L\right)^{-1} 0=0 .
$$

Noticing $u_{1} \in \partial P_{r_{0}}$, we get a contradiction. That is to say,

$$
T u(t) \neq \mu u(t), \quad \forall u \in \partial P_{r_{0}}, \mu \geq 1, t \in[0,1] .
$$

By Lemma 6, we obtain

$$
i\left(T, P_{r_{0}}, P\right)=1 \text {. }
$$

On the other hand, in view of Lemmas 9, 12, and 8, we can choose a constant $R_{0}>r$ such that $i\left(T, P_{R_{0}}, P\right)=1$. Combining this with (86) and (80), we easily get

$$
\begin{aligned}
& i\left(T, P_{R_{0}} \backslash \bar{P}_{r}, P\right)=1, \\
& i\left(T, P_{r} \backslash \bar{P}_{r_{0}}, P\right)=-1 .
\end{aligned}
$$

Hence, BVP (2) has at least two positive solutions.

Theorem 17. Suppose that (H1) and (H2) hold. In addition, suppose that there exist $r_{1}, r_{2}>0$ with $0<r_{1}<r_{2}$ such that

$$
\begin{aligned}
& f(t, u)>m_{0} r_{1}, \quad \forall u \in\left[\frac{M r_{1}}{2}, r_{1}\right], t \in[0,1], \\
& f(t, u)<M_{0} r_{2}, \quad \forall u \in\left[0, r_{2}\right], t \in[0,1],
\end{aligned}
$$

hold, where $m_{0}, M_{0}$ are the same as in Theorems 15 and 16. Then BVP (2) has at least two positive solutions.

Proof. Similar to the proof of Theorem 15, we can choose $0<$ $r_{0}<r_{2}$ such that

$$
\begin{aligned}
& i\left(T, P_{r_{1}}, P\right)=0, \\
& i\left(T, P_{r_{0}}, P\right)=1 .
\end{aligned}
$$

Moreover, for $u \in \partial P_{r_{2}}$, we get

$$
\begin{aligned}
T u(t) & =\int_{0}^{1} G(t, s) f(s, u(s)) d s \\
& <\int_{0}^{1} G(t, s) d s \cdot M_{0} r_{2},
\end{aligned}
$$

which gives $\|T u\|<\max _{t \in[0,1]} \int_{0}^{1} G(t, s) d s \cdot M_{0} r_{2}=r_{2}$. This implies that $T u \neq \mu u$ for all $u \in \partial P_{r_{2}}, \mu \geq 1$. By Lemma 6, we have $i\left(T, P_{r_{2}}, P\right)=1$. This together with (89) guarantees

$$
\begin{aligned}
& i\left(T, P_{r_{2}} \backslash \bar{P}_{r_{1}}, P\right)=1, \\
& i\left(T, P_{r_{1}} \backslash \bar{P}_{r_{0}}, P\right)=-1 .
\end{aligned}
$$

Therefore, BVP (2) has at least two positive solutions.

\section{Examples}

Example 1. Consider the following system:

$$
\begin{aligned}
D_{0^{+}}^{13 / 4} u(t)+\frac{1+t}{3} u e^{u} & =0, \quad t \in(0,1), \\
u(0) & =u^{\prime \prime}(0)=u^{\prime \prime \prime}(0)=0, \\
u^{\prime}(1) & =\frac{1}{2} \int_{0}^{3 / 4} u(s) d s .
\end{aligned}
$$

Then BVP (92) has at least one positive solution.

Proof. BVP (92) can be regarded as a BVP of the form (2), where

$$
f(t, u)=\frac{1+t}{3} u e^{u},
$$

and $\alpha=13 / 4, \lambda=1 / 2, \xi=3 / 4,0 \leq \lambda \xi^{2} \approx 0.28<2$. Obviously, $f_{u}=((1+t) / 3)\left(e^{u}+u e^{u}\right)$, and $f(t, 0)=0, f_{u}(t, 0)=$ $(1 / 3)(1+t)>0, t \in[0,1]$. By direct calculation, we have

$$
\begin{aligned}
& \int_{0}^{1}(1-t)^{\alpha-2} f_{u}(t, 0) d t=\int_{0}^{1}(1-t)^{\alpha-2} \frac{1+t}{3} d t<\frac{2}{3} \\
& \quad<0.97 \approx \frac{\left(1-\lambda \xi^{2} / 2\right) \Gamma(\alpha)}{\alpha-1},
\end{aligned}
$$

and $f_{\infty}=+\infty$. Thus, (H1) and (H2) hold. By Theorem 13, BVP (92) has at least one positive solution.

Example 2. Consider the following system of fractional differential equations:

$$
\begin{aligned}
& D_{0^{+}}^{7 / 2} u(t)+\arctan \left(1+t^{2}\right) \sin ^{2} u \\
& \quad+t e^{t / 2}\left(\frac{1}{3} u+\frac{3+\sqrt{t}}{2}\right)=0, \quad t \in(0,1), \\
& u(0)=u^{\prime \prime}(0)=u^{\prime \prime \prime}(0)=0, \\
& u^{\prime}(1)=3 \int_{0}^{1 / 6} u(s) d s .
\end{aligned}
$$

Then BVP (95) has at least one positive solution. 
Proof. (95) is a BVP of type (2), where

$$
\begin{aligned}
f(t, u)= & \arctan \left(1+t^{2}\right) \sin ^{2} u \\
& +t e^{t / 2}\left(\frac{1}{3} u+\frac{3+\sqrt{t}}{2}\right),
\end{aligned}
$$

and $\alpha=7 / 2, \lambda=3, \xi=1 / 6,0 \leq \lambda \xi^{2} \approx 0.08<2$. By direct calculation, we get that $\lim _{u \rightarrow+\infty}(f(t, u) / u)=$ $(1 / 3) t e^{t / 2}=\phi(t)$ uniformly holds with respect to $t$ on $[0,1]$, $\int_{0}^{1}(1-t)^{\alpha-2} \phi(t) d t<(2 / 3)\left(e^{1 / 2}-1\right)<1.28 \approx(1-$ $\left.\lambda \xi^{2} / 2\right) \Gamma(\alpha) /(\alpha-1)$, and $f_{0}=+\infty$.

Clearly, $f(t, u)$ satisfies conditions (H3). Hence, our conclusion follows from Theorem 14.

Example 3. Consider the following system of fractional differential equations:

$$
\begin{aligned}
& D_{0^{+}}^{13 / 4} u(t)+\frac{\eta}{(2+\sqrt{e})(1+\eta)} u e^{t / 2} \\
& \quad+3600 \sin ^{2} u \ln (3+t)=0, \quad t \in(0,1), \\
& u(0)=u^{\prime \prime}(0)=u^{\prime \prime \prime}(0)=0, \\
& u^{\prime}(1)=\frac{5}{2} \int_{0}^{1 / 5} u(s) d s .
\end{aligned}
$$

Here $\eta$ is a positive constant which satisfies $0<\eta<r^{-1}(L)$. Then BVP (97) has at least two positive solutions.

Proof. We will apply Theorem 15. Corresponding to the problem (2), we take

$$
\begin{aligned}
f(t, u)= & \frac{\eta}{(2+\sqrt{e})(1+\eta)} u e^{t / 2} \\
& +3600 \sin ^{2} u \ln (3+t),
\end{aligned}
$$

and $\alpha=13 / 4, \lambda=5 / 2, \xi=1 / 5,0 \leq \lambda \xi^{2}=0.1<2$.

Obviously, $f_{u}=(\eta /(2+\sqrt{e})(1+\eta)) e^{t / 2}+3600 \sin 2 u \ln (3+$ $t) \in C([0,1] \times[0,+\infty))$, and $f(t, 0)=0, f_{u}(t, 0)=(\eta /(2+$ $\sqrt{e})(1+\eta)) e^{t / 2}>0, t \in[0,1]$. Thus,

$$
\begin{gathered}
\int_{0}^{1}(1-t)^{\alpha-2} f_{u}(t, 0) d t<\frac{\eta}{(2+\sqrt{e})(1+\eta)} \sqrt{e} \\
<\frac{\sqrt{e}}{2+\sqrt{e}}<1.075 \approx \frac{\left(1-\lambda \xi^{2} / 2\right) \Gamma(\alpha)}{\alpha-1},
\end{gathered}
$$

and $f^{\infty}<(\eta /(2+\sqrt{e})(1+\eta)) \sqrt{e}<\eta /(1+\eta)<\eta<r^{-1}(L)$. This means (H1) and (H2) hold.

In addition, by Lemma 5 , we get $m_{0}=\left(\max _{t \in[0,1]} \int_{1 / 2}^{1} G(t\right.$, $s) d s)^{-1} \leq 48.9$. By direct calculation, we have $M=(\alpha-1-$ $\left.\lambda / \alpha-\left(1-\lambda \xi^{2} / 2\right)\right) /(\alpha-1) \approx 0.236, \sin ^{2}(M / 2) \approx 0.014$. Taking $r=1$, we obtain $f(t, u)>3600 \ln (3+t) \sin ^{2}(M / 2)>50.4>$ $m_{0} r$, for any $u \in[0.0118,1]=[(M / 2) r, r]$.

By Theorem 15, system (97) has at least two positive solutions.

\section{Data Availability}

No data were used to support this study.

\section{Conflicts of Interest}

The authors declare that they have no conflicts of interest.

\section{Acknowledgments}

This research was supported by the project of Shandong Province Higher Educational Science and Technology Program of China under grant J18KA233 and the National Natural Science Foundation of China under grant 61873150.

\section{References}

[1] A. A. Leung, "A semilinear reaction-diffusion prey-predator system with nonlinear coupled boundary conditions: equilibrium and stability," Indiana University Mathematics Journal, vol. 31, no. 2, pp. 223-241, 1982.

[2] I. Podlubny, Fractional Differential Equations, Mathematics in Science and Engineering, vol. 198, Academic Press, New York, NY, USA, 1999.

[3] S. G. Samko, A. A. Kilbas, and O. I. Marichev, Fractional Integral And Derivatives (Theory and Applications), Gordon and Breach, Switzerland, 1993.

[4] B. Ahmad and J. J. Nieto, "Existence results for a coupled system of nonlinear fractional differential equations with threepoint boundary conditions," Computers \& Mathematics with Applications, vol. 58, no. 9, pp. 1838-1843, 2009.

[5] B. Ahmad, J. J. Nieto, A. Alsaedi, and M. El-Shahed, "A study of nonlinear Langevin equation involving two fractional orders in different intervals," Nonlinear Analysis: Real World Applications, vol. 13, no. 2, pp. 599-606, 2012.

[6] R. P. Agarwal, N. Hussain, and M. A. Taoudi, "Fixed point theorems in ordered banach spaces and applications to nonlinear integral equations," Abstract and Applied Analysis, vol. 2012, Article ID 245872, 15 pages, 2012.

[7] A. Cabada and G. Wang, "Positive solutions of nonlinear fractional differential equations with integral boundary value conditions," Journal of Mathematical Analysis and Applications, vol. 389, no. 1, pp. 403-411, 2012.

[8] C. S. Goodrich, "Existence of a positive solution to a class of fractional differential equations," Applied Mathematics Letters, vol. 23, no. 9, pp. 1050-1055, 2010.

[9] C. S. Goodrich, "Existence of a positive solution to systems of differential equations of fractional order," Computers \& Mathematics with Applications, vol. 62, no. 3, pp. 1251-1268, 2011.

[10] N. Hussain and M. A. Taoudi, "Krasnosel'skii-type fixed point theorems with applications to Volterra integral equations," Fixed Point Theory and Applications, vol. 2013, no. 1, 16 pages, 2013.

[11] Y. Li, Y. Chen, and I. Podlubny, "Stability of fractional-order nonlinear dynamic systems: Lyapunov direct method and generalized Mittag-Leffler stability," Computers \& Mathematics with Applications, vol. 59, no. 5, pp. 1810-1821, 2010.

[12] J. Mao, Z. Zhao, and N. Xu, "On existence and uniqueness of positive solutions for integral boundary value problems," Electronic Journal of Qualitative Theory of Differential Equations, vol. 16, pp. 1-8, 2010. 
[13] A. S. Perelson, D. E. Kirschner, and R. D. Boer, "Dynamics of HIV infection of $\mathrm{CD}^{+}{ }^{+}$T cells," Mathematical Biosciences, vol. 114, no. 1, pp. 81-125, 1993.

[14] D. Zhao and Y. Liu, "Positive solutions for a class of fractional differential coupled system with integral boundary value conditions," Journal of Nonlinear Science and its Applications. JNSA, vol. 9, no. 5, pp. 2922-2942, 2016.

[15] X. Yang, "Fractional derivatives of constant and variable orders applied to anomalous relaxation models in heat-transfer problems," Thermal Science, pp. 326-326, 2016.

[16] X.-J. Yang, H. M. Srivastava, and J. A. T. Machado, "A new fractional derivative without singular kernel: Application to the modelling of the steady heat flow," Thermal Science, vol. 20, no. 2, pp. 753-756, 2016.

[17] M. Jia and X. Liu, "Multiplicity of solutions for integral boundary value problems of fractional differential equations with upper and lower solutions," Applied Mathematics and Computation, vol. 232, pp. 313-323, 2014.

[18] K. Q. Lan and W. Lin, "Multiple positive solutions of systems of Hammerstein integral equations with applications to fractional differential equations," Journal Of The London Mathematical Society-Second Series, vol. 83, no. 2, pp. 449-469, 2011.

[19] Y. Liu, W. Zhang, and X. Liu, "A sufficient condition for the existence of a positive solution for a nonlinear fractional differential equation with the Riemann-Liouville derivative," Applied Mathematics Letters, vol. 25, no. 11, pp. 1986-1992, 2012.

[20] S. K. Ntouyas, G. Wang, and L. Zhang, "Positive solutions of arbitrary order nonlinear fractional differential equations with advanced arguments," Opuscula Mathematica, vol. 31, no. 3, pp. 433-442, 2011.

[21] M. Stojanovic and R. Gorenflo, "Nonlinear two-term time fractional diffusion-wave problem," Nonlinear Analysis: Real World Applications, vol. 11, no. 5, pp. 3512-3523, 2010.

[22] J. F. Xu, Z. L. Wei, and W. Dong, "Uniqueness of positive solutions for a class of fractional boundary value problems," Applied Mathematics Letters, vol. 25, no. 3, pp. 590-593, 2012.

[23] W. Yang, "Positive solutions for a coupled system of nonlinear fractional differential equations with integral boundary conditions," Computers \& Mathematics with Applications, vol. 63, no. 1, pp. 288-297, 2012.

[24] C. Yuan, "Two positive solutions for (n-1, 1)-type semipositone integral boundary value problems for coupled systems of nonlinear fractional differential equations," Communications in Nonlinear Science and Numerical Simulation, vol. 17, pp. 930942, 2012.

[25] S. Vong, "Positive solutions of singular fractional differential equations with integral boundary conditions," Mathematical and Computer Modelling, vol. 57, no. 5-6, pp. 1053-1059, 2013.

[26] X. Zhang and Y. Han, "Existence and uniqueness of positive solutions for higher order nonlocal fractional differential equations," Applied Mathematics Letters, vol. 25, no. 3, pp. 555-560, 2012.

[27] D. Zhao and Y. Liu, "Twin solutions to semipositone boundary value problems for fractional differential equations with coupled integral boundary conditions," Journal of Nonlinear Sciences and Applications. JNSA, vol. 10, no. 7, pp. 3544-3565, 2017.

[28] H. Zhang, Y. Li, and W. Lu, "Existence and uniqueness of solutions for a coupled system of nonlinear fractional differential equations with fractional integral boundary conditions," Journal of Nonlinear Sciences and Applications. JNSA, vol. 9, no. 5, pp. 2434-2447, 2016.
[29] D. J. Guo and V. Lakshmikantham, Nonlinear Problems in Abstract Cones, Academic Press, San Diego, Calif, USA, 1988.

[30] V. Lakshmikantham and A. S. Vatsala, "Basic theory of fractional differential equations," Nonlinear Analysis: Theory, Methods \& Applications, vol. 69, no. 8, pp. 2677-2682, 2008. 


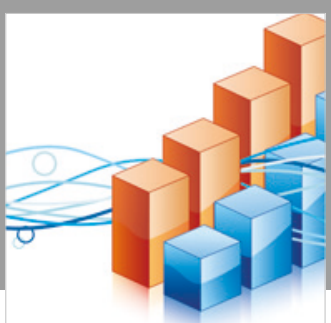

Advances in

Operations Research

\section{-n-m}
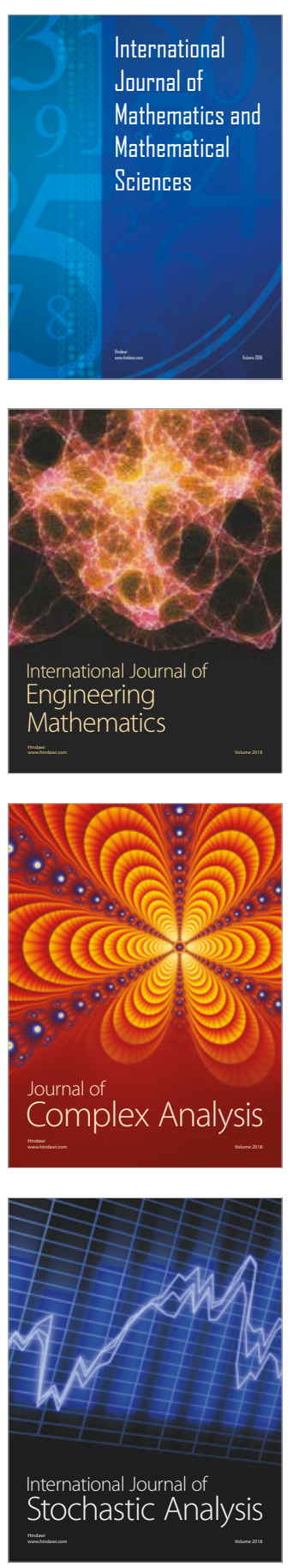
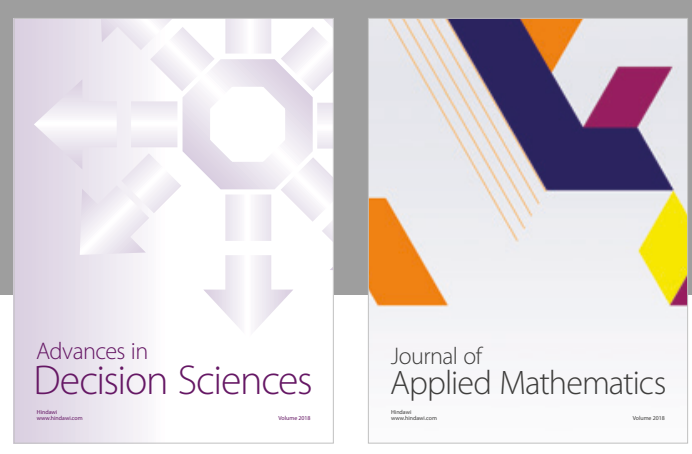

Journal of

Applied Mathematics
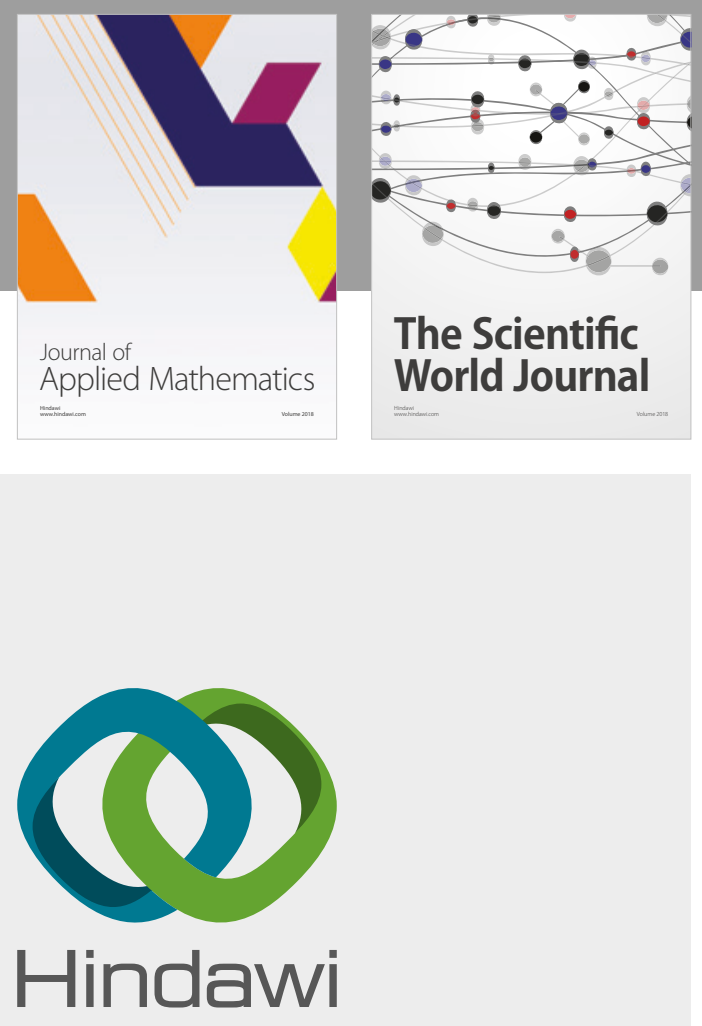

Submit your manuscripts at

www.hindawi.com

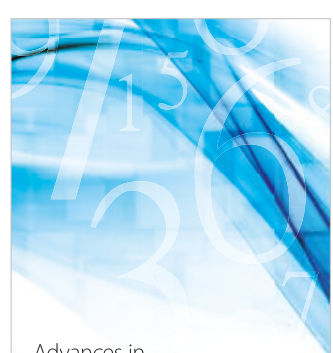

Advances in
Numerical Analysis
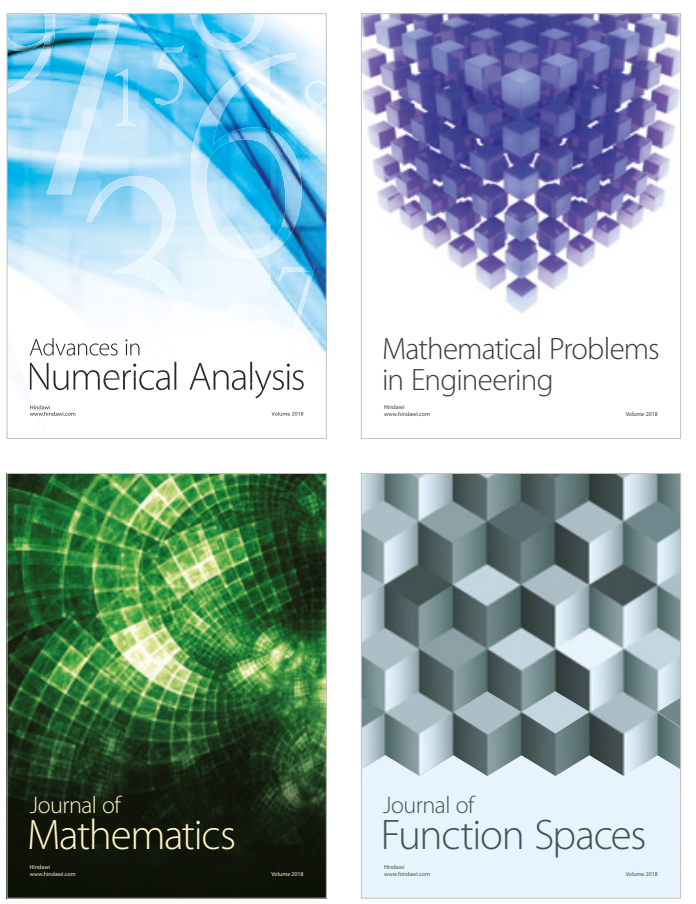

Mathematical Problems in Engineering

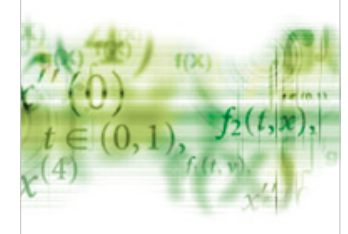

International Journal of

Differential Equations

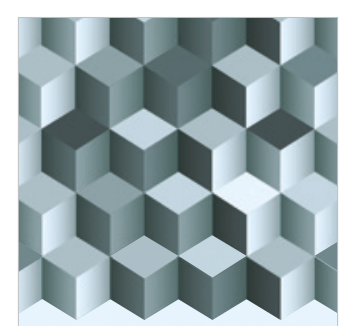

Journal of

Function Spaces

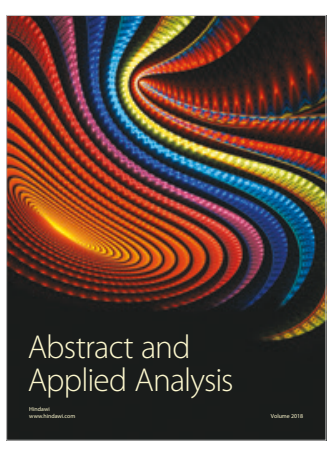

The Scientific

World Journal

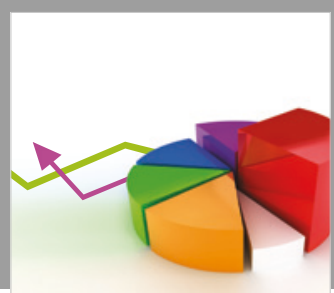

Journal of

Probability and Statistics
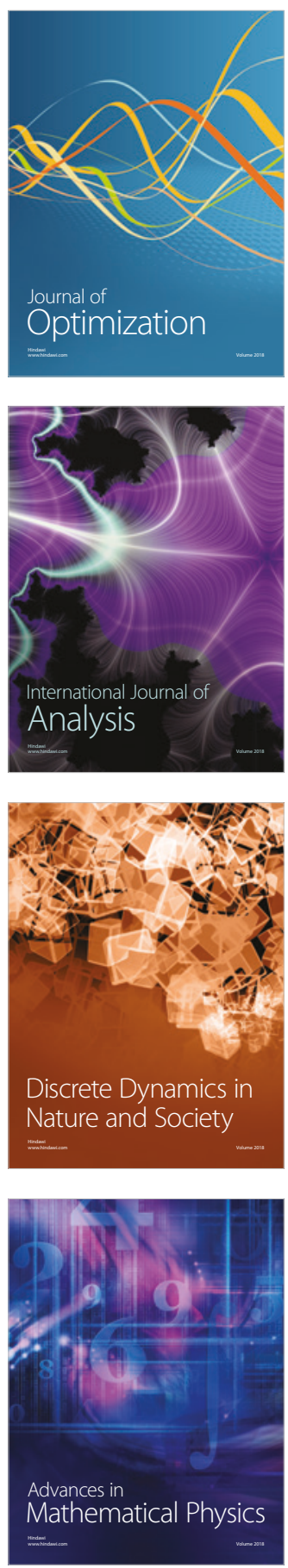